\title{
Age and Body Make a Difference in Optimistic Health Beliefs and Nutrition Behaviors
}

\author{
Britta Renner, Nina Knoll, and Ralf Schwarzer
}

\begin{abstract}
Nutrition behaviors are governed by health beliefs such as risk perceptions, outcome expectancies, and optimistic self-beliefs. This study deals with the role that objective criteria such as age and body weight might play in forming subjective beliefs. The question is whether they can deter people from forming an overly optimistic judgment of their health risk. Six kinds of verbal judgments were assessed, namely self-reported health, vulnerability toward cardiovascular diseases, nutrition outcome expectancies, nutrition self-efficacy, intentions to change one's diet, and reported nutrition behaviors. In a sample of 1,583 men and women between 14 and 87 years of age, these judgments were statistically related to age and body weight. It was found that people do take their objective risk status into account, but only to a certain degree. The self-serving bias continues to exist throughout all age groups and weight levels. Moreover, it was found that individuals report better intentions to adhere to healthy foods and better nutrition behaviors as they grow older and gain weight.
\end{abstract}

Key words: age, body weight, nutrition, self-efficacy, optimism

Weight control and preventive nutrition are considered to be important health behaviors that people should maintain, along with physical activity, to attain health, longevity, and fitness. Following a healthy diet, low in saturated fat and high in fiber, is a popular medical recommendation. According to present medical knowledge, such nutrition helps to prevent cardiovascular disease and other ailments.

Britta Renner, Institut für Psychologie, Ernst-Moritz-Arndt Universität Greifswald, Greifswald, Germany; Nina Knoll and Ralf Schwarzer, Gesundheitpsychologie, Freie Universität Berlin, Berlin, Germany.

Correspondence concerning this article should be addressed to Britta Renner, Psychologie, Ernst-Moritz-Arndt Universität Greifswald, Franz-Mehring-Strasse 47, 17487 Greifswald, Germany. E-mail: renner@mail.uni-greifswald.de 
However, most individuals do not adhere to this health behavior, and many have not even contemplated adopting it (Ogden, 1996). The Berlin Risk Appraisal and Health Motivation Study was launched to examine the sociocognitive factors of health behavior change in various domains, such as physical exercise, smoking, alcohol consumption, and preventive nutrition. In this analysis, the focus will be on the latter, examining to what degree nutrition-related beliefs are based on objective health indicators such as age and body weight.

Objective health status is related to age because people are more prone to degenerative diseases and multimorbidity with increasing age (Steinhagen-Thiessen \& Borchelt, 1999). Also, objective health status is related to body weight because overweight individuals are at a higher risk of suffering from cardiovascular and other diseases (e.g., Assmann, 1993; Bray, 1978; WHO Study Group, 1990). Therefore, health beliefs should account for this continuous decline and disadvantage in some way. Conversely, it has been found that people do not picture the actual risk they are in, although they do understand that others are at risk. They often think, "A negative event may happen to others, but it won't happen to me." For example, asking people how they would judge their risk of having a heart attack, compared to that of an average person of the same sex and similar age (the "average" risk), typically yields a "below average" estimate (e.g., Hahn \& Renner, 1998). This bias in risk perception, which has been coined unrealistic optimism or optimistic bias (Perloff \& Fetzer, 1986; Weinstein, 1980), reflects the difference between the perceived risk of oneself and that of others within the same reference group and belongs to the broader construct of defensive optimism (Schwarzer, 1994). Weinstein (1996) reviewed about 200 empirical studies devoted to the issue of unrealistic optimism. However, most of these studies were based on college students or young adults, which raises the question of whether defensive optimism may be restricted to adolescents and young adults, groups that may be especially prone to unrealistic optimism, as suggested by some developmental researchers (Arnett, 1995; Elkind, 1974). One could argue that adolescents as well as young adults display unrealistic optimism because they have not experienced a decline in health. This line of reasoning has received indirect support from empirical findings reported by Weinstein $(1980,1982,1987)$. He found that unrealistic optimism decreased with illness experience. He argued that in the absence of past illness experience, people may mistakenly conclude that they are exempt from future risk. Following this line of reasoning, the elderly should make more realistic inferences from their illnesses or those of their age group and consequently judge their own vulnerability in the same manner as that of their peers. Conversely, one could argue that defensive optimism holds up as a life-long motivational tendency to protect oneself from threatening thoughts and, by this, to shield mental health (Taylor \& Brown, 1988). There is no clear empirical evidence to support this assumption but it is supported indirectly by research on well-being. For example, Staats et al. (1993) reported that optimistic perceptions of age and future quality of life are 
maintained in older persons. Furthermore, recent studies have documented that older persons are not unhappier than middle-aged or younger persons (e.g., Filipp, 1996; Mroczek \& Kolarz, 1998). Following this theoretical position, one would expect a systematic underestimation of one's own health risk as compared to that of others in any age group.

Furthermore, to more fully elucidate the relation between age and optimistic health beliefs, it is important to take other objective characteristics, in addition to age, into account. In the context of nutrition-related beliefs, body weight is certainly an influential variable. Overweight individuals are expected to take their disadvantage into account when making health-related judgments. Thus, they should consider themselves as being at higher risk than people of average weight. But, on the other hand, they might still persist in distorted social comparisons, displaying unrealistic optimism. Apparently, there are no previous findings of an interaction between age and objective risk status characteristics such as body weight. This leads to the question of whether body weight adds explanatory value over and beyond the influence of age. For example, one could assume that people display a tendency for self-defensive compensation, relying more on positive health characteristics while discounting negative ones.

To initiate and maintain health behaviors, it is not sufficient to perceive a serious risk for oneself. In addition, one should also perceive positive action-outcome contingencies and believe in one's own capability to perform the intended behavior (Schwarzer \& Fuchs, 1996; Schwarzer \& Renner, in press). This leads to alternative conceptions of optimism that are adaptive rather than defensive in nature because they imply instrumental coping behaviors. This functional optimism (Schwarzer, 1994; Taylor, 1989) includes positive outcome expectancies, but also personal resources, such as perceived self-efficacy (Bandura, 1997). Outcome expectancies (e.g., "If I stick to a low-fat diet, then I will reduce my risk of suffering a heart attack") can be the most influential beliefs in the motivation to change nutrition behavior (Schwarzer, 1992, 1999). Perceived self-efficacy (e.g., "I can manage to stick to healthy food even if something delicious but unhealthy is served") portrays individuals' beliefs in their capabilities to exercise control over challenging demands and over their own functioning. Such optimistic self-beliefs shape the goals people set for themselves, what courses of action they choose to pursue, how much effort they invest in given endeavors, and how long they persevere in the face of barriers and setbacks. These beliefs do not simply reflect the present coping competencies of an individual but slightly overestimate them to become effective. It is assumed that defensive and functional optimism share a common ground, such as the motive to protect or enhance self-esteem. Therefore, individuals may display defensive as well as functional optimism. One could reason that poorer health due to aging or risk factors such as being overweight will lead to a reduction of defensive and functional optimism: One feels more at risk and becomes doubtful of one's capabil- 
ity of performing the intended health behavior. On the other hand, aging is associated with more experience in managing daily activities as well as initiating behavior changes despite various obstacles. This suggests that despite failing health, individuals may become more capable of coping with obstructions in order to maintain optimistic self-beliefs. Hence, people may acknowledge their health risks with increasing age or body weight but would still regard their personal coping resources in an optimistic way.

This study attempts to elaborate on aging, body weight, and optimistic health beliefs. The research question is to what degree individuals realistically consider their age and body weight when making judgments about their health risks, personal resources, and health behaviors. Initially, it examines whether young adults are more likely than the elderly to overstate their own invulnerability to harm (defensive optimism) and their personal coping resources (functional optimism). Secondly, it questions whether body weight is associated with both forms of optimism. And finally, is considers whether body weight adds explanatory value over and beyond the influence of age.

\section{METHOD}

\section{Procedure}

Sixty six percent of the participants were recruited through advertisements placed in local newspapers in Berlin, Germany. In addition, a letter describing the study was sent to people insured with the Technician's Health Insurance Agency (Techniker Krankenkasse) who lived near the four study locations (two universities and two city halls). Upon arrival, they were informed that the study was part of a community-wide effort to collect health information from the population at various locations of the city. Those who agreed to participate completed a brief questionnaire that included five items assessing subjective health and risk perception. Afterwards, they were thanked and received a more detailed questionnaire that included 20 items assessing positive outcome expectancies, perceived self-efficacy, behavioral intentions, and nutrition behavior. This one was completed at home and sent back in an enclosed envelope.

\section{Measures}

Self-reported health status. The following question was asked to assess subjective health status: "Would you say your health in general is excellent, very good, good, fair, or poor?" This measure was formulated in line with the health status question of the National Health Interview Survey (Schechter, 1993). 
Risk perception. Risk estimates were obtained for each of the following four cardiovascular diseases: hypertension, heart disease, stroke, and heart attack. To assess the perceived absolute own risk, participants were asked to estimate the likelihood of experiencing each particular health problem, for example, "How high do you think is your risk of becoming hypertensive during your life time?" (see Perloff \& Fetzer, 1986). The same question was asked for an average target person of one's own age and sex (absolute other's risk; e.g., "How likely do you think is it that someone else of your sex and age will become hypertensive during his or her life time?"). Responses were made on 7-point Likert scales ranging from -3 (extremely unlikely) to 3 (extremely likely).

Positive outcome expectancies were measured by seven items. Participants were asked: "What do you think will be the consequences for yourself if you adopt a low-fat diet?" Following this header, responses were elicited to seven more specific questions: "If I stick to a low-fat diet, then ..." (a) "I will feel physically more attractive," (b) "I will feel better mentally," (c) "I will have no (or fewer) body weight problems," (d) "I will lower my cholesterol level," (e) "I will lower my blood pressure," (f) "I will be healthier," and (g) "I will reduce my risk of suffering a heart attack." Responses were made on 4-point scales ranging from 1 (strongly disagree) to 4 (strongly agree). Internal consistency was satisfactory with Cronbach's $\alpha=.84$.

For the assessment of perceived self-efficacy, four different items were used (Cronbach's $\alpha=.74)$. The general stem for all items was, "How certain are you about being able to overcome the following barriers? I can manage to stick to healthy food ...." Afterwards, specific barriers were presented: (a) "even if something delicious but unhealthy is served," (b) "even if this will be more expensive," (c) "even if I am short of time to do my shopping and preparation," and (d) "even if this means I cannot eat everything I crave for." Responses were made on 4-point scales ranging from 1 (strongly disagree) to 4 (strongly agree).

The intention to adopt healthy nutrition behaviors was measured with four items, namely (a) "I intend to eat only a very low amount of fat (such as animal fat, cheese, butter) over the next months," (b) "I intend to live a healthier life," (c) "I intend to eat healthy foods over the next months," and (d) "I intend to invest more into my health." Responses were made on 7-point scales ranging from 1 ("I don't have this intention at all") to 7 ("I do have this intention"). Cronbach's alpha was considerably high, with $\alpha=.89$.

Nutrition behavior was assessed with four items related to a low-fat diet: (a) "I follow a low-fat diet," (b) "When I eat milk products or drink milk, I choose low-fat products (such as low-fat milk or yogurt)," (c) "When I eat cheese, I choose high-fat products (such as Gouda or Emmentaler)," and (d) "I avoid foods with cholesterol." Responses were made on 4-point scales ranging from 1 (strongly disagree) to 4 (strongly agree). The negatively poled item was recoded before an overall score was created (Cronbach's $\alpha=.74$ ). 
Weighing, height measures, and Body Mass Index (BMI). Weighing and height measures were standardized (calibrated scales, regular clothing). The BMI was calculated as the weight in kilograms divided by the square of the height in meters. According to international standards (Bailey \& Ferro-Luzzi, 1995; Bray, 1978; WHO Study Group, 1990), participants with a BMI over 24 were classified as being overweight, whereas those with a BMI below this cutoff point were classified as being of average weight.

\section{Sample}

A total of 1,583 inhabitants of Berlin between 14 and 87 years of age volunteered to participate in the study. Seventy-one percent of the participants $(n=1,019)$ completed both the first and the second questionnaire.

\section{RESULTS}

\section{Details of the Resulting Sample}

Participants recruited through advertisements were slightly heavier than those who were invited through a personal letter, with a mean BMI of $25.1(S D=3.7)$ versus $24.3(S D=3.6) ; t(1,1581)=3.11, \mathrm{p}=.002$. Furthermore, gender differences between the two groups were significant, $\chi^{2}(1)=43.7, p<.001$. Women participants were mostly recruited through advertisements, whereas men participants were predominantly motivated by the personal letter. Age differences among the two groups, however, were not significant, $t(1,1581)=1.52, p=.129$.

Dropout analysis showed that those who completed both questionnaires were on average 4 years older than those who did not; 40 years $(S D=15.3)$ versus 36 years $(S D=14.0), t(1,581)=5.38, p<.001$. Furthermore, they exhibited a slightly but significantly higher BMI than the dropout group, 24.6 $(S D=3.6)$ versus $23.9(S D=3.4)$; $t(1,581)=3.74, p<.001$. There was no significant gender difference between the two samples, $\chi^{2}(1)=.33, p=.56$. All subsequent analyses are based on the reduced sample of $n=1,019$ participants who had completed both questionnaires.

Of this final sample, 53\% were women and $47 \%$ were men, $\chi^{2}(1)=4.40, p=$ .035. As Table 1 shows, $53 \%$ were younger than 41 years of age, and $61 \%$ had a BMI lower than or equal to 24 .

\section{Self-Reported Health}

As expected, the percentage of participants reporting excellent or very good health status declined steadily with increasing age (Figure 1). About $65 \%$ of the younger participants (younger than 31 years of age) reported their overall health as being 
TABLE 1

Absolute and Relative Frequencies for "Age Group" and "Body Weight" (Longitudinal Sample)

\begin{tabular}{lcccccc}
\hline & $\begin{array}{c}\text { Younger } \\
\text { Than 31 }\end{array}$ & $31-40$ & $40-50$ & $51-60$ & $\begin{array}{c}\text { Older } \\
\text { Than 60 }\end{array}$ & Total \\
\hline Average weight & 328 & 111 & 66 & 75 & 36 & 616 \\
BMI < 25 (\%) & 32 & 11 & 7 & 7 & 4 & 61 \\
Overweight & 43 & 62 & 96 & 112 & 90 & 403 \\
BMI $\geq 25(\%)$ & 4 & 6 & 9 & 11 & 9 & 39 \\
Total & 371 & 173 & 162 & 187 & 126 & 1,019 \\
& $(37 \%)$ & $(17 \%)$ & $(16 \%)$ & $(18 \%)$ & $(12 \%)$ & $(100 \%)$ \\
\hline
\end{tabular}

Note. Twelve individuals were older than 74 years; BMI = Body Mass Index.

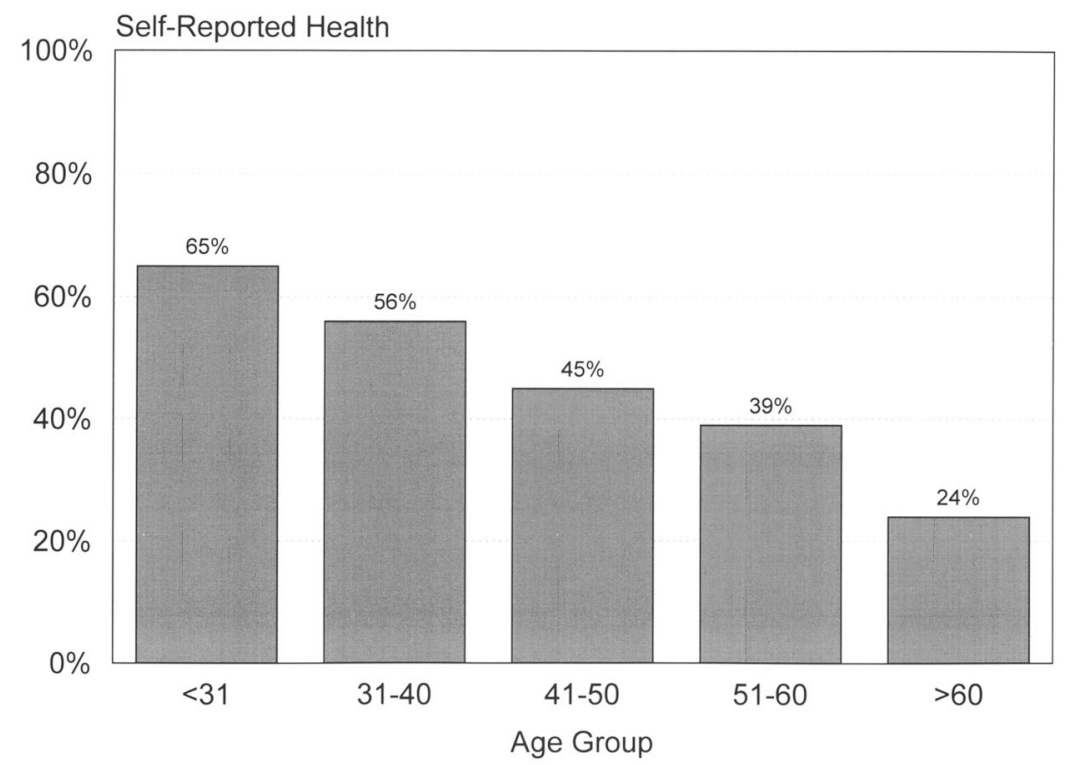

FIGURE 1 Very good or excellent self-reported health by age group.

very good or excellent. In contrast, only $24 \%$ of the age group 61 or older stated that they were in very good health, $\chi^{2}(1)=62.22, p<.001$. Women and men were as likely as to claim that their health was very good or excellent, $49 \%$ versus $52 \%$; $\chi^{2}(1)=0.16, p=.690$. In addition, participants seemed to take their risk status into account. Those with average weight were more likely to report very good or excellent health than those who were overweight. Only $38 \%$ of the overweight respondents said that their health was excellent or very good, whereas $58 \%$ of the partici- 
pants with average weight viewed their health as excellent or very good, $\chi^{2}(1)=$ $37.30, p<.001$. In conclusion, participants obviously considered objective factors such as age and body weight when judging their overall health status. These results are remarkably consistent with the existing survey literature on self-reported health status (e.g., Siegel, 1994) and corroborate reports of similar associations between self-reported health status, gender, age, and BMI.

According to these results, aging and being overweight are associated with a decrease in subjective health. Does this mean that aging and being overweight are also associated with an increase in perceived risk, followed by a decline in defensive optimism? The ensuing question is whether perceived vulnerability to cardiovascular diseases is also associated with age and risk status. A $2 \times 5 \times 2 \times 2$ mixed model multivariate analysis of variance was conducted, with the two risk judgments (self vs. other) as levels of the within-subjects factor "Target." The five age groups (younger than 31, 31-40, 41-50, 51-60, older than 60), gender (men vs. women), and weight group (average weight vs. overweight) constituted the levels of the three between-subjects factors. ${ }^{1}$ The analyses yielded a significant main effect for the within-factor "Target," $F(1,999)=57.75, p<.001$. Figure 2 displays the mean absolute risk judgments for self and others separately for each age group. Subsequent analysis showed that self-ratings increased with age, $F(4,999)=$ $13.34, p<.001$, as well as absolute risk ratings for an average peer of the same age and sex, $F(4,999)=4.71, p=.001$.

Hence, personal risk perceptions reveal relative accuracy, because older participants gave higher risk ratings than younger ones. However, at the same time, evidence for defensive optimism emerged. Participants expected comparatively more negative outcomes to happen to other people than to themselves. Further analysis showed a significant discrepancy between self-ratings and ratings for an average member of the same age group and gender within each age group, all $F(1,999)$ values greater than 9 , with $p<.001$. The observed effect of aging on perceived risk leads to the conclusion that people acknowledged a higher risk with increasing age and declining health. Nevertheless, they still assumed that they were less vulnerable than their peers. In other words, aging did not curb unrealistic optimistic risk perceptions.

\footnotetext{
${ }^{1}$ All the figures and reported results are based on full factorial models that meet standard requirements of analysis of variance (ANOVA). The effects were estimated by the regression method for partition sums of squares. That means that each effect is adjusted for all the other effects in the model. For analyzing risk perceptions, a mixed between-/within-subjects ANOVA had been used. Therefore, the total sum of squares (SS) is divided into a source attributable to the three between-subjects factors of the design (body weight, age, and sex), and a source attributable to the within-subjects factor named "Target" (risk perception for oneself and for an average peer). In addition, factor level differences were examined by constructing simple main effects that mean to compute the effect of one factor within the level of another factor (e.g., the effect of age within overweight participants).
} 


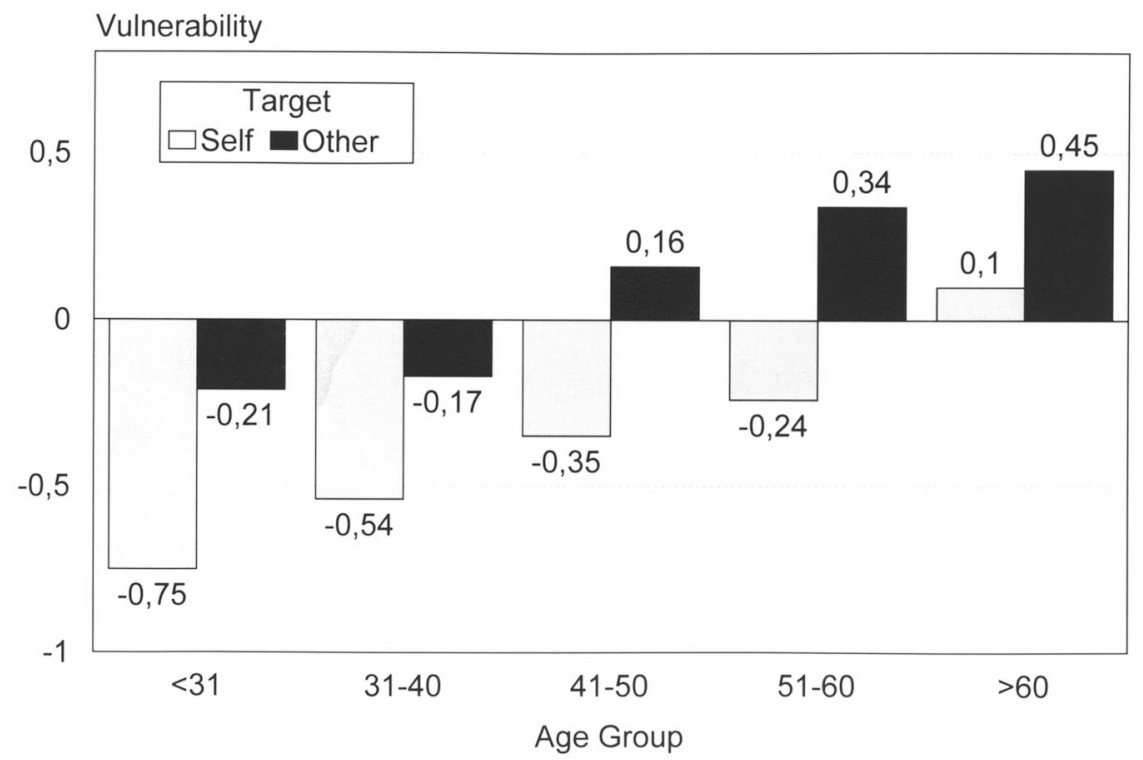

FIGURE 2 Mean absolute risk judgments for self and others to cardiovascular diseases as a function of age.

Moreover, participants of average weight considered themselves at lower risk than overweight participants, -.67 versus $-.12 ; F(1,999)=10.30, p=.001$. With respect to the assumed risk faced by an average peer, -.05 versus .16; $F(1,999)=$ $0.11, p=.74$, both weight groups displayed similar ratings. Comparing the mean judgment for the self and the average peer revealed a significant unrealistic optimism for the average weight group, $F(1,999)=139.13, p<.001$, and for the overweight group, $F(1,999)=18.54, p<.001$.

Finally, compared to men, women showed a lower risk perception for themselves, -.67 versus $-.20 ; F(1,999)=12.98, p<.001$. However, they did not differ in the perception of an average peer, -.06 versus .08; $F(1,999)=0.06, p=.81$. Nevertheless, men, $F(1,999)=29.35, p<.001$, as well as women, $F(1,999)=$ $124.91, p<.001$, displayed unrealistic optimism.

These result patterns further support the assumption that people consider their actual risk status when judging their vulnerability: Older and overweight participants and men admitted that they were at greater risk, but even so they still considered themselves as less vulnerable than an average peer. Hence, people take their age and risk status into account when gauging their risk, but this realism is still accompanied by defensive optimism: One feels less vulnerable than others. This leads to the question of whether there is a similar relation between functional optimism (positive outcome expectancies and perceived self-efficacy), aging, and being overweight. 


\section{Positive Outcome Expectancies}

The $2 \times 2 \times 5$ ANOVA yielded a significant main effect for age, $F(4,999)=4.20, p$ $=.002$; weight, $F(1,999)=30.12, p<.001$; and gender, $F(1,999)=15.82, p<.001$. Furthermore, the two main effects of age and body weight were qualified by a significant interaction, $F(4,999)=2.53, p=.04$. Overall, the effects accounted jointly for $12 \%$ of the variation in outcome expectancies.

Compared to men, women displayed more positive outcome expectancies, followed by adopting a healthy diet (23.3 vs. 22.0). Moreover, as Figure 3 displays, overweight participants harbored more positive outcome expectancies in comparison to average weight participants within each age group. Even at young age, overweight participants anticipated clear positive outcomes for themselves. Hence, within the overweight group, aging was not associated with a gain of expected benefit from a healthy diet, $F(4,999)=0.59, p=.67$. In contrast, the analysis within the average weight group revealed a significant positive trend for outcome expectancies with increasing age, $F(4,999)=6.01, p<.001$. Additionally, Scheffé post hoc contrasts within the average weight group showed that individuals aged 50 years or younger exhibited significantly less positive outcome expectancies than older ones.

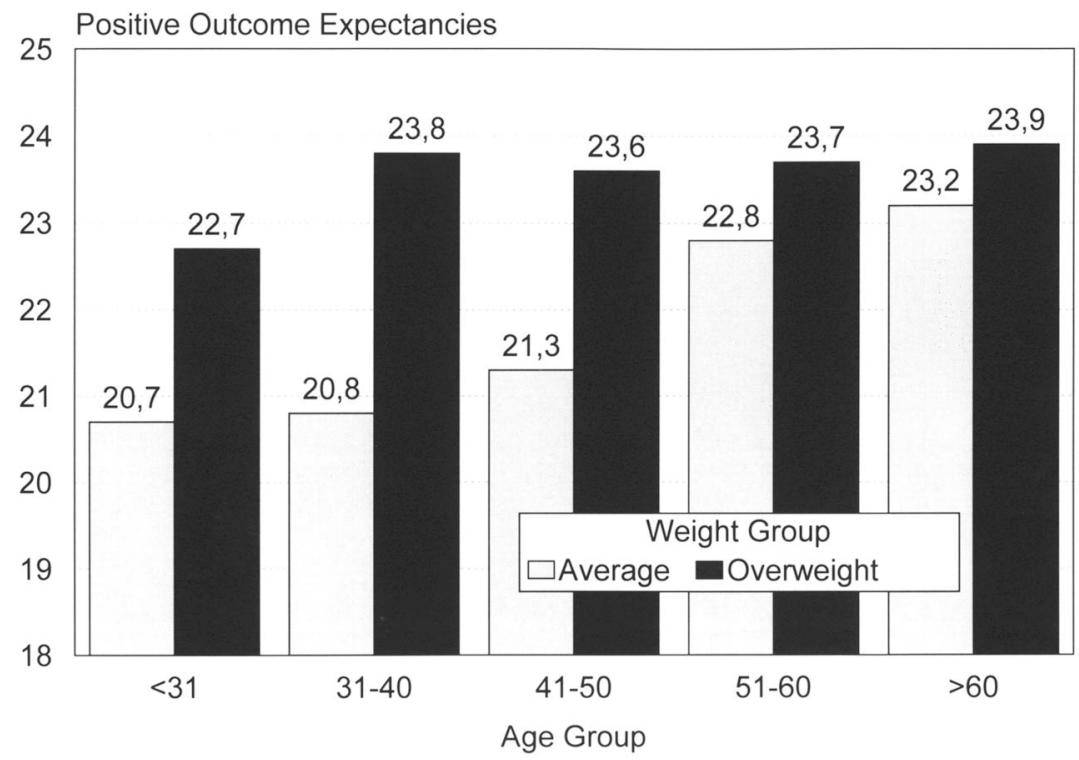

FIGURE 3 Mean positive outcome expectancies as a function of age and weight. 


\section{Perceived Self-Efficacy}

The $2 \times 2 \times 5$ ANOVA yielded a significant main effect for age, $F(4,999)=21.78$, $p<.001$, and a significant interaction between age and weight group, $F(4,999)=$ $3.92, p=.004$. No other effect reached statistical significance. Overall, $11 \%$ of the self-efficacy variance was accounted for by these two effects.

In accordance with the previous results, older participants perceived higher self-efficacy than younger ones. This holds for average weight, $F(4,999)=15.40$, $p<.001$, as well as overweight participants, $F(4,999)=8.25, p<.001$. As Figure 4 displays, within both groups there was a visible positive linear trend for perceived self-efficacy with increasing age. However, within the overweight group the effect is more pronounced. Scheffé post hoc contrast analysis within overweight participants showed that (with only a few exceptions) an increase of self-efficacy from the youngest to the oldest group took place among the average participants. However, Scheffé post hoc contrast analysis within average weight participants confirmed this trend only partially. Up to the age of 50 there was no significant increase in self-efficacy (all Scheffé post hoc contrasts with $p>.05$ ). But beyond this age, self-efficacy increased with age.

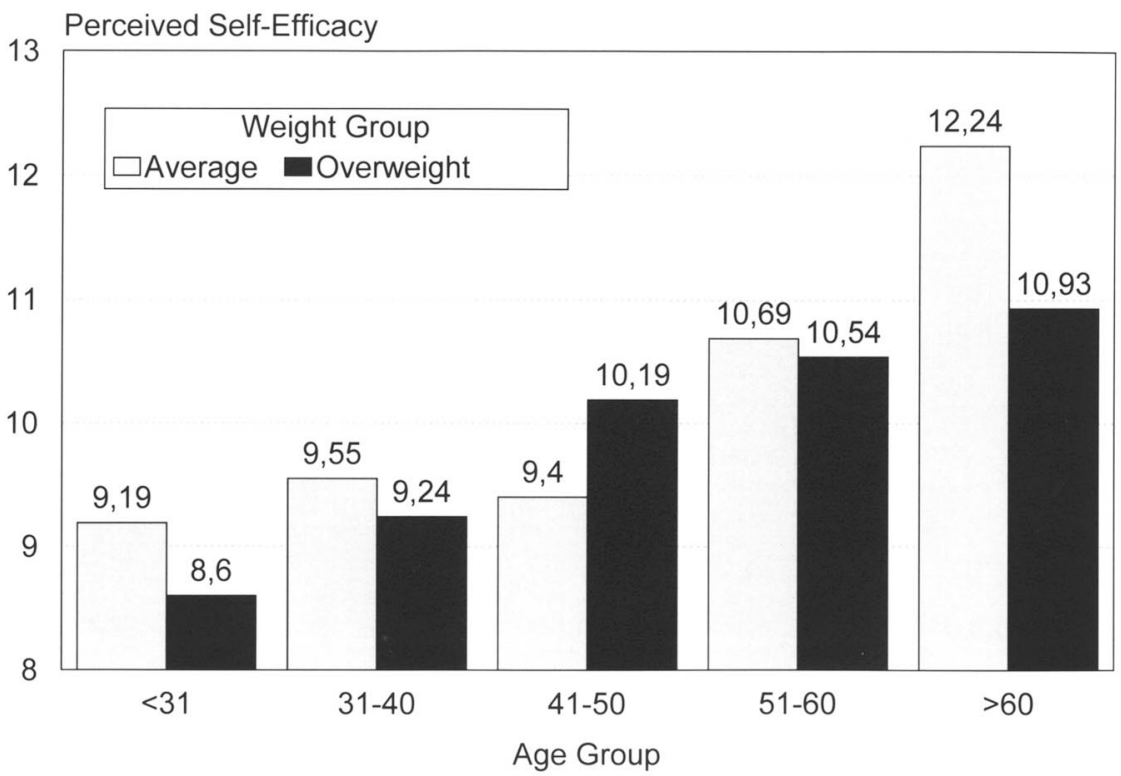

FIGURE 4 Mean perceived self-efficacy as a function of age and weight. 


\section{Intention to Adopt Low-Fat Nutrition}

There was a significant main effect for age, $F(4,999)=10.55, p<.001$; body weight, $F(1,999)=9.44, p=.002$; and gender, $F(1,999)=10.36, p=.001$. In addition, an interaction between age and body weight, $F(4,999)=3.60, p<.01$, reached significance. Eleven percent of the variation of the intention to adopt low-fat nutrition was accounted for by these effects.

Women reported a higher intention to adopt low-fat nutrition compared to men (20.4 vs. 19.4). Older as well as overweight participants reported higher intentions to alter their eating habits according to a low-fat diet than younger ones or participants with average weight.

Closer inspection of the interaction effect between age and weight revealed a stronger linear effect of age within participants of average weight, $F(4,999)=9.09, p$ $<.01$, as compared to overweight participants, $F(4,999)=3.28, p=.01$. Figure 5 shows that among average weight participants, intentions for preventive nutrition behaviors increased with age. However, this positive trend was only partly supported by Scheffé post hoc contrasts. Participants younger than 50 years reported lower intentions than older ones. Younger overweight participants showed a similar positive trend, but the differences were smaller. Therefore, Scheffé post hoc contrasts only upheld the notion that, when compared to the older-than-60 group, the youngest group had lower intentions to adopt a low-fat nutrition.

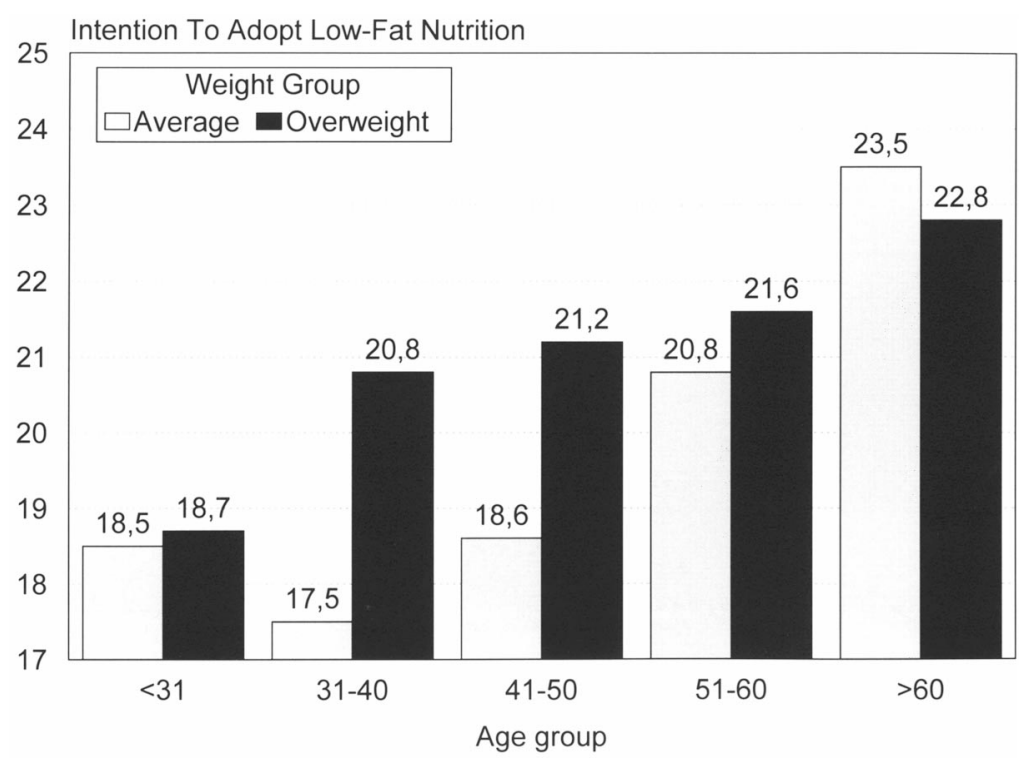

FIGURE 5 Mean intention to adopt a low-fat diet as a function of age and weight group. 


\section{Preventive Nutrition}

As before, the $2 \times 2 \times 5$ ANOVA yielded a significant main effect for age, $F(4,999)$ $=30.70, p<.001$; body weight, $F(1,999)=4.65, p=.03$; and gender, $F(1,999)=$ $17.50, p<.001$. Furthermore, an interaction between age and body weight, $F(4$, $999)=6.75, p<.001$, emerged. These four effects accounted together for $21 \%$ of the preventive nutrition variance.

Compared to men, women reported healthier nutrition behaviors (10.4 vs. 11.3). Overweight participants reported a healthier diet than average weighted participants, and aging was associated with healthier nutrition habits. Figure 6 shows the mean consumption of healthy foods as a function of age and body weight.

The positive effect of aging on nutrition could be observed within the average weight group, $F(4,999)=29.66, p<.001$, as well as within overweight participants, $F(4,999)=10.73, p<.001$. Scheffé post hoc contrasts within average weight participants supported the finding that aging secured preventive nutrition. For overweight individuals, the effect was somewhat weaker. Therefore, Scheffé post hoc contrasts suggest that mainly the youngest and the oldest age groups differed significantly from the remaining groups.

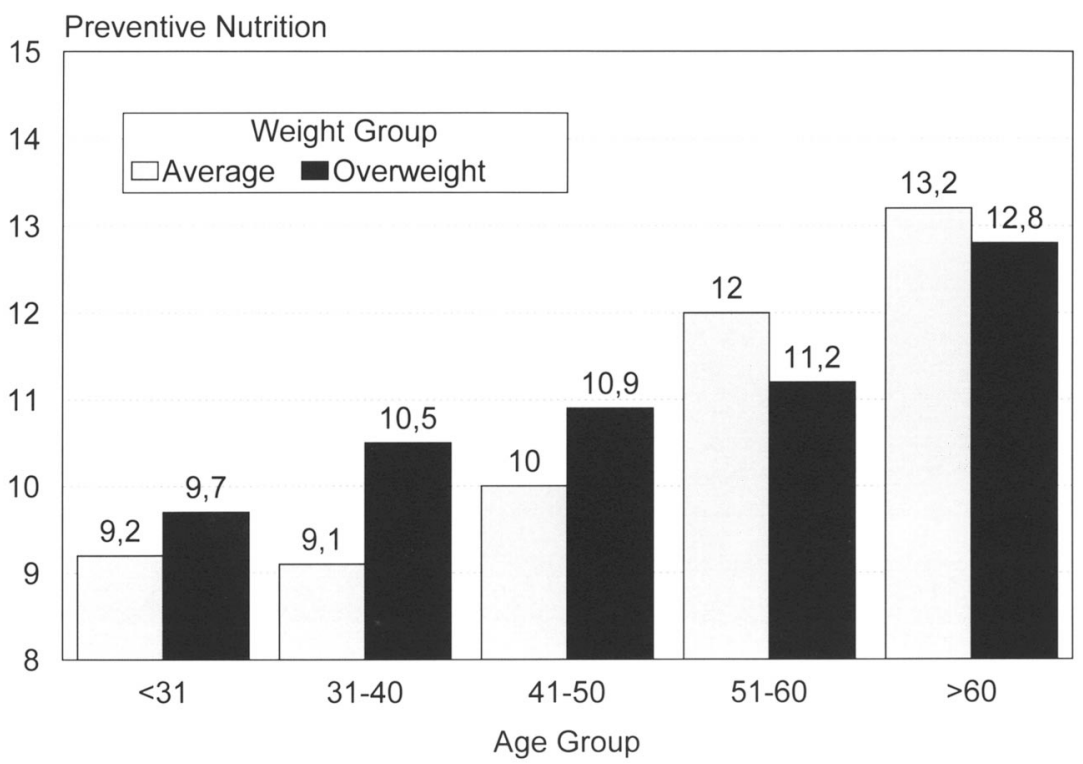

FIGURE 6 Mean consumption of low-fat food as a function of age and weight group. 


\section{DISCUSSION}

The results have documented important associations between two objective health-related factors and six kinds of health beliefs that deal with preventive nutrition. People take their age and body weight into account when they judge their health status. On average, the older and the heavier they are, the less frequently they regard their health as "very good or excellent" (see Figure 1). This is realistic and not surprising. It also demonstrates that individuals do not merely make judgments in terms of social comparisons within a narrow reference group of similar peers, but that they use overarching criteria, for instance, objective health status or comparisons across the life-span.

The same realism is expressed when people gauge their vulnerability toward cardiovascular health threats. The older they become, the higher they calibrate their risk (see Figure 2).

However, the most interesting aspect is the phenomenon of how they deal with their reference group. Within each age group, they discount their risk compared to that of others. This demonstrates the existence of an optimistic bias throughout life, although participants were not indiscriminately optimistic, and tended to adhere partly to the constraints of reality. The results suggest that the strong defensive optimism effect was determined by the tendency to see others as more at risk for negative events than oneself. The fact that individuals harbor pessimistic biases for others may represent a mechanism by which they maintain a comparatively optimistic outlook for themselves, despite realizing that health-related risks do increase with age. This might satisfy a need for accuracy by acknowledging more objective risk at an absolute level, but serve self-protective needs by maintaining a pessimistic view of others at the same time (Armor \& Taylor, 1998; Whitley \& Hern, 1991). The social network, be it real or imagined, can be seen as a convoy that moves through the life course and serves as a backdrop for self-serving social comparisons.

Relative invulnerability may not only be achieved by adapting average risk to one's own risk status. Overweight participants showed higher risk perceptions for themselves than average weight participants, but they did not differ with respect to their perceived average risk. Because both groups displayed unrealistic optimism, defensive optimism appears to be a matter of degree. Similar findings were obtained in a study among college students (Rothman, Klein, \& Weinstein, 1996). People take their objective disadvantages into account when making judgments, but they discount them to some extent. This leads to a practical conclusion: Risk communication that provides only information about the individual's risk may have less impact in comparison to a risk communication that provides additional information about the risk faced by an average peer. People may need both kinds of information to locate their risk status more accurately.

An important question is whether defensive optimism poses a barrier against the adoption of indispensable health behaviors. Why should someone adopt a diffi- 
cult behavior, such as restrained eating, when no risk is perceived in comparison to others? Again, this is a matter of degree. In this sample, increasing age and body weight are indeed related to risk perception, although they are slightly discounted. The other health beliefs that were assessed in this study document an inclination toward preventive nutrition. This is, for example, expressed in increased consumption of low-fat food with advancing age in both weight groups (see Figure 6), which is in line with three related health beliefs, namely (a) nutrition outcome expectancies (Figure 3), (b) nutrition self-efficacy (Figure 4), and (c) the intention to consume low-fat foods (Figure 5). This is cumulative evidence for risk-related reasonable health behavior. Delving further into these data, it was discovered that age and functional optimism were more closely interrelated within average weight participants. With advancing age, they acknowledged more positive consequences and greater confidence to carry out the intended behavior, expressed higher intentions to adopt a low-fat nutrition, and displayed healthier nutrition habits. In overweight participants, there was a less positive trend associated with age. To some extent this could be due to a ceiling effect because even young overweight participants exhibited a high level of positive outcome expectancies and intention to stick to a healthy nutrition. This may reflect greater self-relevance and vulnerability within overweight young people. In conclusion, these differential effects indicate that the participants were at different points in the behavior change process depending on their objective risk status. Hence, different interventions and information may be needed to move people closer to action. Older and overweight people may benefit less from risk communication than from resource communication, emphasizing the effectiveness of precautions, and their coping abilities.

On the other hand, health risk perception is nothing but a starting point for health behavior change. It sets the stage for subsequent cognitions such as outcome expectancies and self-efficacy, while individuals progress through a motivational phase that concludes with an explicit behavioral intention (e.g., the intention to consume low-fat, high-fiber foods). In the subsequent postintentional phase, they plan and execute the critical behavior with different degrees of success. This view has been put forward in the Health Action Process Approach (Schwarzer, 1992, 1999) and other process theories of health behavior change.

These findings shed some light on the changes in optimistic health beliefs brought on by the aging process, but several caveats must be considered in interpreting the findings. First, cross-sectional data do not allow the examination of an aging effect as opposed to a cohort effect. It may be that the current older generation of adults harbors greater optimism than future generations. Second, the results may stem from a self-selection bias. It is possible that older men and women with a more pessimistic view of their health-related capabilities did not volunteer to participate in the study. This would have led to a sampling bias. Another possibility is that people with a more pessimistic self-view may be at greater risk for serious health problems, making morbidity and mortality more likely. This would create a 
group of survivors within the older age strata with higher levels of optimism than those in the younger strata. If so, levels of functional optimism in samples of older people may be higher simply through attrition of pessimistic people. To determine whether the present findings reflect a genuine aging phenomenon, a sample would have to be followed longitudinally from youth to old age. Only such a prospective study across the life-span would allow a thorough examination of the underlying processes of health belief changes.

\section{REFERENCES}

Armor, D. A., \& Taylor, S. E. (1998). Situated optimism: Specific expectancies and self-regulation. In M. P. Zanna (Ed.), Advances in experimental social psychology (Vol. 30, pp. 309-379). New York: Academic.

Arnett, J. (1995). The young and the reckless: Adolescent reckless behavior. Current Directions in Psychological Science, 4, 67-71.

Assmann, G. (1993). Lipid metabolism disorders and coronary heart disease. Primary prevention, diagnosis and therapy guidelines for general practice (2nd ed.). München, Germany: MMV Medizin Verlag.

Bailey, K. V., \& Ferro-Luzzi, A. (1995). Use of body mass index of adults in assessing individual and community nutritional status. WHO Bulletin, 73, 673-680.

Bandura, A. (1997). Self-efficacy: The exercise of control. New York: Freeman.

Bray, G. (1978). Definition, measurement, and classification of the syndromes of obesity. International Journal of Obesity, 2, 99-112.

Elkind, D. (1974). Children and adolescents: Interpretative essays on Jean Piaget. New York: Oxford University Press.

Filipp, S.-H. (1996). Motivation and emotion. In J. E. Birren \& K. W. Schaie (Eds.), Handbook of the psychology of aging (4th ed., pp. 218-235). San Diego, CA: Academic.

Hahn, A., \& Renner, B. (1998). Perception of health risks: How smoker status affects defensive optimism. Anxiety, Stress, and Coping, 11, 93-112.

Mroczek, D. K., \& Kolarz, C. M. (1998). The effect of age on positive and negative affect: A developmental perspective on happiness. Journal of Personality and Social Psychology, 75, 1333-1349.

Ogden, J. (1996). Health psychology. Buckingham, England: Open University Press.

Perloff, L. S., \& Fetzer, B. K. (1986). Self-other judgments and perceived vulnerability to victimization. Journal of Personality and Social Psychology, 50, 502-510.

Rothman, A. J., Klein, W. M., \& Weinstein, N. D. (1996). Absolute and relative biases in estimations of personal risk. Journal of Applied and Social Psychology, 26, 1213-1236.

Schechter, S. (1993). Investigation into the cognitive processes of answering self-assessed health status questions (Cognitive Methods Staff, Working Paper Series, No. 2, Office of Research and Methodology). Hyattsville, MD: National Center for Health Statistics.

Schwarzer, R. (1992). Self-efficacy in the adoption and maintenance of health behaviors: Theoretical approaches and a new model. In R. Schwarzer (Ed.), Self-efficacy: Thought control of action (pp. 217-242). Washington, DC: Hemisphere.

Schwarzer, R. (1994). Optimism, vulnerability, and self-beliefs as health-related cognitions: A systematic overview. Psychology and Health, 9, 161-180.

Schwarzer, R. (1999). Self-regulatory processes in the adoption and maintenance of health behaviors. The role of optimism, goals, and threats. Journal of Health Psychology, 4, 115-127. 
Schwarzer, R., \& Fuchs, R. (1996). Self-efficacy and health behaviors. In M. Conner \& P. Norman (Eds.), Predicting health behaviour: Research and practice with social cognition models (pp. 163-196). Buckingham, England: Open University Press.

Schwarzer, R., \& Renner, B. (in press). Perceived self-efficacy governing preventive nutrition: A longitudinal study of a community sample. Health Psychology.

Siegel, P.Z. (1994). Self-reported health status: Public health surveillance and small-area analysis. In S. Schechter (Ed.), Proceedings of the 1993 NCHS Conference on the cognitive aspects of self-reported health status (pp. 11-27; Cognitive Methods Staff, Working Paper Series, No. 10. Office of Research and Methodology). Hyattsville, MD: National Center for Health Statistics.

Staats, S., Heaphey, K., Miller, D., Partlo, C. I., Romine, N., \& Stubbs, K. (1993). Subjective age and health perceptions of older persons: Maintaining the youthful bias in sickness and in health. International Journal of Aging and Human Development, 37, 191-203.

Steinhagen-Thiessen, E., \& Borchelt, M. (1999). Morbidity, medication, and functional limitations in very old age. In P. B. Baltes \& K. U. Mayer (Eds.), The Berlin aging study: Aging from 70 to 100 (pp. 131-166). New York: Cambridge University Press.

Taylor, S. E. (1989). Positive illusions: Creative self-deception and the healthy mind. New York: Basic Books.

Taylor, S. E., \& Brown, J. D. (1988). Illusion and well-being: A social psychological perspective on mental health. Psychological Bulletin, 103, 193-210.

Weinstein, N. D. (1980). Unrealistic optimism about future life events. Journal of Personality and Social Psychology, 39, 806-820.

Weinstein, N. D. (1982). Unrealistic optimism about susceptibility to health problems. Journal of Behavioral Medicine, 5, 441-460.

Weinstein, N. D. (1987). Unrealistic optimism about susceptibility to health problems: Conclusions from a community-wide sample. Journal of Behavioral Medicine, 10, 481-500.

Weinstein, N. D. (1996). References on perceived invulnerability and optimistic biases about risk or future life events. Unpublished manuscript.

Whitley, B. E., \& Hern, A. L. (1991). Perceptions of vulnerability to pregnancy and the use of effective contraception. Personality and Social Psychology Bulletin, 17, 104-110.

WHO Study Group. (1990). Diet, nutrition, and the prevention of chronic diseases (World Health Organization Tech. Rep. Series 797). Geneva, Switzerland: World Health Organization. 